\title{
Tracking On-Surface Chemistry with Atomic Precision
}

\author{
Peter H. Jacobse*a,b (D) \\ Marc-Etienne Moret ${ }^{a}$ (i) \\ Robertus J. M. Klein Gebbink ${ }^{\mathrm{a}}$ \\ Ingmar Swart ${ }^{b}$ \\ a Organic Chemistry and Catalysis, Debye Institute for \\ Nanomaterials Science, Utrecht University, Universiteits- \\ weg 99, 3584 CG, Utrecht, The Netherlands \\ p.h.jacobse@uu.nl \\ ${ }^{b}$ Condensed Matter and Interfaces, Debye Institute for \\ Nanomaterials Science, Utrecht University, PO Box \\ 80000, 3508 TA Utrecht, The Netherlands
}

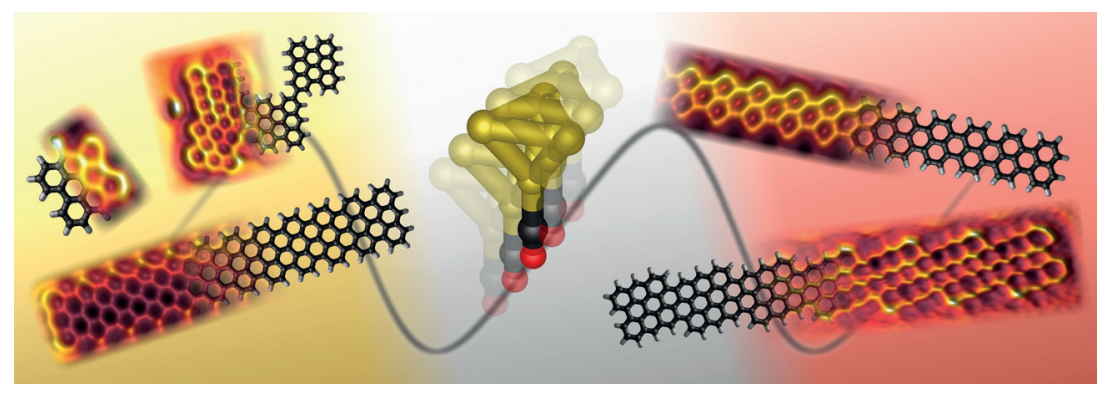

Received: 02.06.2017

Accepted after revision: 14.07.2017

Published online: 17.08 .2017

DOI: 10.1055/s-0036-1590867; Art ID: st-2017-p0425-sp

Abstract The field of on-surface synthesis has seen a tremendous development in the past decade as an exciting new methodology towards atomically well-defined nanostructures. A strong driving force in this respect is its inherent compatibility with scanning probe techniques, which allows one to 'view' the reactants and products at the singlemolecule level. In this article, we review the ability of noncontact atomic force microscopy to study on-surface chemical reactions with atomic precision. We highlight recent advances in using noncontact atomic force microscopy to obtain mechanistic insight into reactions and focus on the recently elaborated mechanisms in the formation of different types of graphene nanoribbons.

Key words on-surface synthesis, atomic force microscopy, polycyclic aromatic hydrocarbons, graphene nanoribbons, single molecule

Surface chemistry has played a prominent role in organic synthesis for centuries, most notably in the form of heterogeneous catalysis. The discovery of platinum-catalyzed combustion of gaseous mixtures by Davy ${ }^{1}$ was only the beginning of a rich history of surface chemistry that would prove to have a tremendous impact on chemistry at large. Although the chemical transformations themselves take place at the surface, the way in which heterogeneous catalysis is employed is most often three-dimensional, with the catalyst dispersed in the reaction medium or the medium being passed through a catalyst bed. Furthermore, the rugged and often ill-defined surface of the catalyst can hardly be considered two-dimensional. With the advent of on-surface synthesis, however, reactions have started to become truly two-dimensional. Here, molecules are deposited onto an atomically flat surface under typically ultrahigh vacuum (UHV) conditions. The reactants can move laterally over the surface but have no freedom to 'jump off' the surface: this would imply desorption, which, under UHV conditions, is

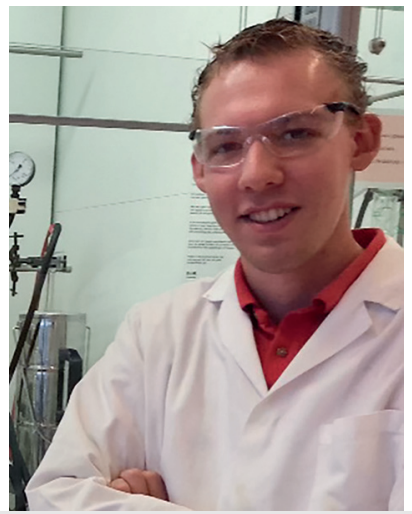

Peter Jacobse was born in Emmen and raised near the city of Groningen, the Netherlands. He studied chemistry and physics at the University of Groningen, and after completing his BSc (2012), he moved to Utrecht to complete his studies in the field of nanomaterials science. He obtained an MSc (Honors, cum laude) from Utrecht University in 2014 under the supervision of Dr. Ingmar Swart. He wrote a research proposal about advancing the study of graphene nanoribbons, for which he was awarded a grant from the Debye Institute for Nanomaterials Science at Utrecht University, where he works to this day.

permanent. Reactions between molecules therefore take place in-plane. The rules of the game of on-surface synthesis therefore allow for a distinct type of chemistry that facilitates the controlled synthesis of new types of nanostructures. Another powerful aspect of this technique is its synergy with conventional chemical methods: The precursor molecules for on-surface reactions can be prepared by solution-based methods before being transferred to the UHV setup. Indeed, on-surface reactions can be applied to a large proportion of molecules that can be tailored in the chemistry lab. Therefore, on-surface synthesis can be particularly powerful when used in conjunction with solution-phase synthesis. 
In on-surface synthesis, precursor molecules are commonly deposited onto a surface by evaporation from an effusion cell followed by adsorption, after which heating or irradiation may be carried out to induce chemical reactions. These reactions may be intramolecular, but in the quest for extended nanostructures, intermolecular coupling reactions are particularly desirable. On-surface chemistry has proven to be a valuable technique to obtain high-quality one- or two-dimensional materials like graphenes, polyphenylenes, fullerenes, and graphene nanoribbons (GNRs). ${ }^{2-8}$ Restriction of adsorbates to the surface effectively eliminates nonselective out-of-plane reactions, facilitating the creation of atomically well-defined low-dimensional nanostructures.

Despite its virtues, on-surface synthesis could only take off as a new emerging field with the advent of sensitive surface-probing techniques. Traditional chemical synthesis mostly relies on spectroscopic techniques and other bulk characterization methods, which are ill-suited for the typically obtained submonolayer coverages on single crystals. Therefore, it was not until the development of highly sensitive methods such as scanning probe microscopy that onsurface chemistry could really develop into a field of its own.

The most used scanning probe technique in on-surface chemistry is scanning tunneling microscopy. Here, a bias voltage is applied between a conductive tip and sample. When the tip-sample distance is on the order of a (few) nanometer(s), electrons can tunnel across the vacuum gap between tip and surface, creating a current which may be used as a feedback parameter to control the tip height. ${ }^{9}$ When scanning the tip across a surface littered with molecular adsorbates, the tunnel current depends on both the physical height as well as the available electronic energy levels that may contribute to the tunneling process. An STM topograph of an absorbed species may thus be regarded as a convolution of its geometric and electronic structure. As far as electronic structure is concerned, STM probes the lowenergy states, which in large aromatic systems, such as polycyclic aromatic hydrocarbons (PAHs) and nanographenes, typically arises from $\pi$-orbitals. STM is a powerful technique in identifying molecules and nanostructures on surfaces, and has the additional advantage that the bias voltage can be used as an extra degree of freedom to facilitate a detailed electronic analysis. ${ }^{10}$ However, the pure geometric structure - the framework of bonds and atoms cannot be identified as it is clouded by electrons from frontier orbitals, as well as non-resonant tunneling processes.

In contrast, noncontact atomic force microscopy is a more recently established technique - and admittedly, a much more demanding one - that is capable of imaging the chemical structure of molecules with atomic resolution. ${ }^{11}$ In nc-AFM, an AFM cantilever with high stiffness (typically
$1,800 \mathrm{~N} \mathrm{~m}^{-1}$ ) is driven at its resonance frequency. Upon approaching the tip to the surface, the resonance frequency shifts due to electrostatic, van der Waals, and Pauli interactions with the sample. This landscape of interactions is established by the total electron density, rather than the resonant electron density, and therefore includes all covalent bonds. As a result, an nc-AFM scan can actually uncover the molecular framework of bonds and atoms, and allows to peek 'inside' a single molecule. ${ }^{12}$ The remarkable capability of nc-AFM to really reveal the molecular 'skeleton' with atomic resolution is unprecedented.

The first realization of intramolecular contrast with ncAFM came with the seminal visualization of the individual benzene rings in the backbone of pentacene. ${ }^{11}$ Soon after, a plethora of other, mostly aromatic molecules were examined, each time revealing images of their respective backbones of bonds and rings in a way that closely resembled the structural models from chemistry textbooks. ${ }^{13-19}$ Noteworthy is the extensive effort in elucidating the structure of asphaltene molecules. ${ }^{20}$ Like snowflakes, no two of these large PAHs are alike, and therefore, their structure cannot be identified with ensemble techniques. On the contrary, by using nc-AFM, each single asphaltene could be identified by the atoms and rings displayed in the respective images.

Nc-AFM is a powerful tool to uncover the structure of known or unknown molecules and becomes even more interesting when the molecules themselves engage in chemistry. Reactions on surfaces can be induced by means of heating, ${ }^{2,3,21,22}$ irradiation, ${ }^{23}$ or even by employing voltage pulses with an STM tip. ${ }^{24,25}$ In each case, nc-AFM can be used to directly compare the structure of the molecular adsorbates before and after the transformation. The unique insight that this provides is particularly useful in reactions with multiple steps, multiple products, or products that cannot be unambiguously identified with other methods.

A beautiful example of a reaction with multiple steps is the tip-induced debromination and reversible Bergman cyclization of 9,10-dibromoanthracene. ${ }^{26}$ By application of a voltage pulse, Schuler et al. succeeded in selectively cleaving off the bromine atoms, yielding the 9-bromo-10-anthryl radical and subsequently the 9,10-anthrylene diradical. Both the reactant and the two individual radicals were visualized with nc-AFM, as can be seen in Figure 1 (a). Surprisingly, it was found that further application of voltage pulses induced reversible switching of the molecule into three different isomeric states. The isomers turned out to be the diradical and two equivalent retro-Bergman products, with the outermost ring cleaved internally to give an expanded, ten-membered ring. By using nc-AFM, the team was able to visualize the ring-expanded molecules, clearly revealing the cleaved bond and giving indisputable evidence of the transformation. 

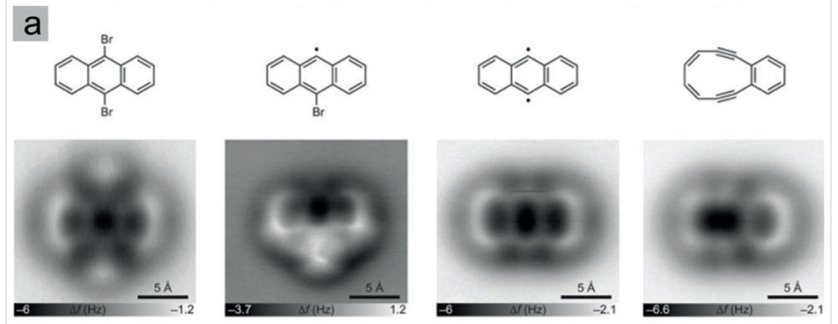

b
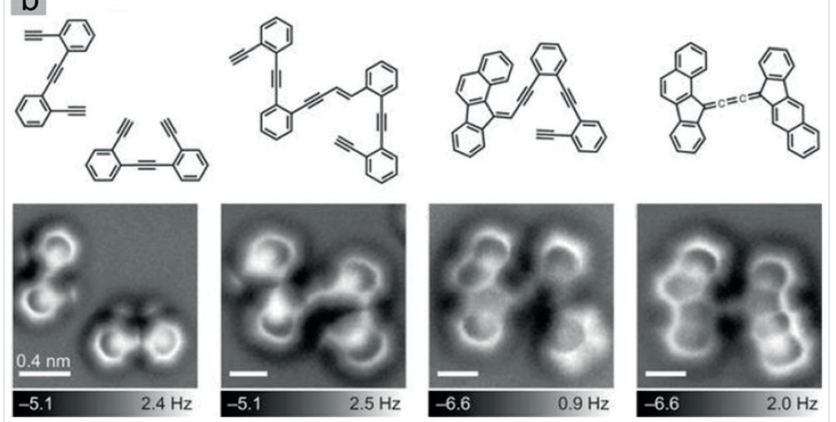

Figure 1 a) Products and intermediates of the Bergman cyclization, identified with nc-AFM. Adapted by permission from Macmillan Publishers Ltd: Nature Communications, ref. 26, Copyright (2016). b) Coupling and cyclization reactions of oligo-(phenylene-1,2,-ethynylenes). Adapted by permission from Macmillan Publishers Ltd: Nature Chemistry, ref. 27, Copyright (2016).

Other elegant examples of isomers and intramolecular transformations analyzed with nc-AFM include the subtle differentiation of cumulene and aryne tautomers in a $\mathrm{PAH},{ }^{28}$ cyclization of triangular dehydrobenzoannulene, ${ }^{22}$ reactions of oligo-(phenylene-1,2-ethynylenes) ) $^{27,29}$ (see Figure $1, \mathrm{~b}$ ), sulfur elimination from diphenanthrothiophene, ${ }^{30}$ and conformational changes in tetraphenylporphyrins ${ }^{31}$ and dibenzo $[a, h]$ thianthrene. ${ }^{32}$ The last three examples show that nc-AFM can even be used on molecules that are not completely planar, although at the expense of losing contrast in regions that are closer to the surface. All examples mentioned above are elegant instances of using AFM in visualizing different products, reactants, and intermediates within single molecules. In the rest of this article, we will review its application to intermolecular reactions.

In the context of intermolecular on-surface reactions tracked with AFM, the dimerization and polymerization of enediynes, ${ }^{29}$ the formation of a metal-organic framework from 4,9-diaminoperylene-quinone-3,10-diimine, ${ }^{33}$ and coupling of porphine onto a graphene sheet ${ }^{34}$ are noteworthy examples. Coupling reactions also play an important role in the bottom-up assembly of potentially extremely useful nanostructures: GNRs. GNRs are particularly promising structures as they are based on the parent material graphene, which features unprecedented electronic properties such as high-charge carrier mobility and low effective mass. Their one-dimensional, quantum-confined nature allows for additional advantageous properties such as a finite and tunable band gap, where fine-tuning of the electronic structure can be achieved through tailoring the width, edge structure, and doping. ${ }^{35-45}$ As such, they hold promise for use in future nanoelectronics. In their pioneering work, Cai et al. presented the first bottom-up synthesis of atomically well-defined GNR on surface. ${ }^{46}$ The ribbons were fabricated in a two-step process from 10,10'-dibromo-9,9'-bianthryl (DBBA) on an $\mathrm{Au}(111)$ surface. As shown in Figure 2 (a), the first step is a thermally induced debromination, giving rise to surface-stabilized radicals that couple to form staggered polyanthrylene chains. ${ }^{47-49}$ The subsequent annealing step<smiles>Brc1c2ccccc2c(-c2c3ccccc3c(Br)c3ccccc23)c2ccccc12</smiles><smiles>Cc1c2ccccc2c(-c2c3ccccc3c(C)c3ccccc23)c2ccccc12</smiles>
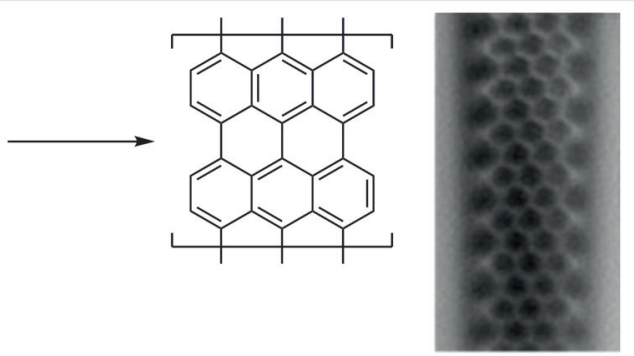<smiles>BrC1=C2C=CC=CC2(B2c3ccccc3B(c3c4ccccc4c(Br)c4ccccc34)C3C=CC=CC23)c2c1cc(Br)c1ccccc21</smiles>

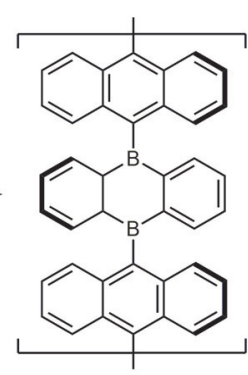
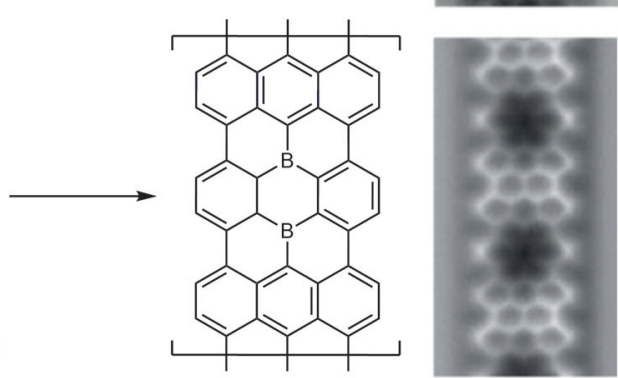

Figure 2 a) Synthesis of 7-AGNR from DBBA. b) Synthesis of boron-doped 7-AGNR from 9,10' '-dibromo-9',10'-diborateranthryl. Adapted by permission from Macmillan Publishers Ltd: Nature Communications, refs. 50, 56. Copyright $(2013,2015)$. 
takes place at higher temperature and induces cyclodehydrogenation of the polyanthrylene into seven-atom wide nanoribbons with armchair-type edges (7-AGNR). Indeed, STM images obtained from samples prepared with omission of the annealing step show a characteristic pattern of protrusions as expected from the staggered anthryl units in polyanthrylene chains. Furthermore, the 7-AGNR were unambiguously resolved as such with nc-AFM (see Figure 2, a).$^{50}$ In the subsequent development of more types of nanoribbons, on-surface synthesis has played a dominant role. ${ }^{51}$ Not only does it facilitate the fabrication of atomically defect-free structures that are difficult to obtain with solution-based methods, but is also inherently compatible with scanning probe techniques that allow for detailed characterization. ${ }^{4,5,52-55}$

Further tailoring of the electronic properties of graphene nanoribbons can be performed by making use of heteroatom doping. For example, nitrogen can be incorporated by substituting phenyl groups with pyridyl groups in the precursor molecules. ${ }^{57-59}$ Both Cloke et al. and Kawaii et al. showed that the bottom-up assembly could also be used to produce boron-doped 7-AGNR, by making use of a precursor that can be thought of as a functionalized analogue of DBBA. ${ }^{56,60}$ This molecule has a single diboraanthracene moiety sandwiched between two bromoanthryl extremities (Figure 2, b). The radical coupling takes place at the bromoanthryl groups in the same exact way as in DBBA, and may thus be thought to be 'blind' for the embedded diboraanthracene. However, when the subsequent cyclodehydrogenation step forces the 'previously unnoticed' boron-substituted anthryl units to enter the lattice, it is not difficult to notice an analogy with the Trojan horse. Nc-AFM images of the product show the same structure as the undoped 7AGNR, but with characteristic variations in $\sigma$-bond contrast due to the substitutional boron atoms.

The boron-doped 7-AGNR is just one of many different GNRs that were produced by cleverly designing and modifying precursor molecules. ${ }^{57,61-63}$ The preparation requires, in each case, a number of solution-phase synthesis steps. Importantly, aryl-aryl coupling reactions similar to those used on surface may already play an important role in the fume hood, before the molecules are ready to enter the UHV setup. For example, DBBA itself has been produced by Kumada and Suzuki cross-coupling reactions of anthryl bromides. ${ }^{50,64}$ Suzuki coupling has also been used in the preparation of the precursors for the 13-atom-wide GNR, by attaching biphenylboronic acid and thiophenylphenylboronic acid onto the bianthryl core. ${ }^{61,65}$ In the latter case, a tetrabromo coupling partner was used, but fortunately the coupling turned out to be selective towards the desired disubstituted product. The Ullmann coupling on the surface, on the other hand, is a homocoupling, and does not permit the same level of selectivity as can be achieved in solution. These results highlight the importance to control the different possible coupling steps, so that they can be carried out in the right sequence. Since the large majority of aryl-aryl cross-coupling reactions rely on aryl halides, the greatest synthetic flexibility can in principle be achieved by carrying out the on-surface coupling with the most strongly bound halogen. This recognition led us to pursue the question whether aryl chlorides could be used instead of aryl bromides in the synthesis of GNR. ${ }^{64}$

We started by synthesizing 10,10'-dichloro-9,9'-dibromo-bianthryl (DCBA): the dichloro analogue of DBBA. Interestingly, when thermally treated in a similar way as with DBBA, DCBA was found to give rise to extensive planar aromatic networks, rather than 7-AGNRs. Nc-AFM clearly revealed these networks to consist of randomly interconnected bisanthene units (bisanthene being the cyclodehydrogenation product of bianthryl; see Figure 3). To figure out why these disordered structures were obtained, we tried to identify mechanistic intermediates by conducting the experiment at a range of different temperatures. At $200{ }^{\circ} \mathrm{C}$, we observed a large number of bisanthene molecules as well as coupled bisanthenes. Once again, we used nc-AFM to reveal their structure, allowing us to discover that the coupling products almost exclusively feature interconnecting bonds from the 'middle' $\mathrm{C}(10)$ position to the 'corner' $\mathrm{CH}(3)$ position, as can be seen in Figure 3 (where we use the numbering of the parent bianthryl molecules). What could be the reason that the usual radical-radical coupling is blocked, and intercepted by this alternative pathway?

The final piece of the puzzle was found by preparing a sample at the even lower temperature of $120^{\circ} \mathrm{C}$, where the vast majority of DCBA molecules remain unaffected and only a few undergo chemical transformations. Instead of coupling together, we found isolated molecules of monochlorobisanthene (see Figure 3), with the chlorine clearly distinguishable from the nc-AFM images. These images proved that cyclodehydrogenation can actually preceed dechlorination, effectively reversing the order of Ullmann coupling and cyclodehydrogenation as compared to DBBA. This result can be ascribed to the increased aryl halide bond strength in DCBA compared to DBBA, causing the dehalogenation temperature to surpass the cyclodehydrogenation temperature. Since the radicals in the reaction mechanism are planar bisanthene radicals, rather than the staggered bianthryl radicals, the natural coupling behavior is suddenly severely impeded by steric effects. The least sterically hindered position, the corner, then remains as the most likely candidate for coupling, and indeed, bisanthene radicals couple radical-to-corner, or from $\mathrm{CH}(3)$ to $\mathrm{C}(10)$. In conclusion, although we established that GNRs could not be obtained from DCBA, our analysis of mechanistic intermediates using nc-AFM allowed us to fully reveal their behavior on a surface.

Another reaction mechanism that we were interested in was the formation of nanoribbons from bianthryls on $\mathrm{Cu}(111)$. Intuitively, one may think that the Ullmann coupling and cyclodehydrogenation should take place in the 


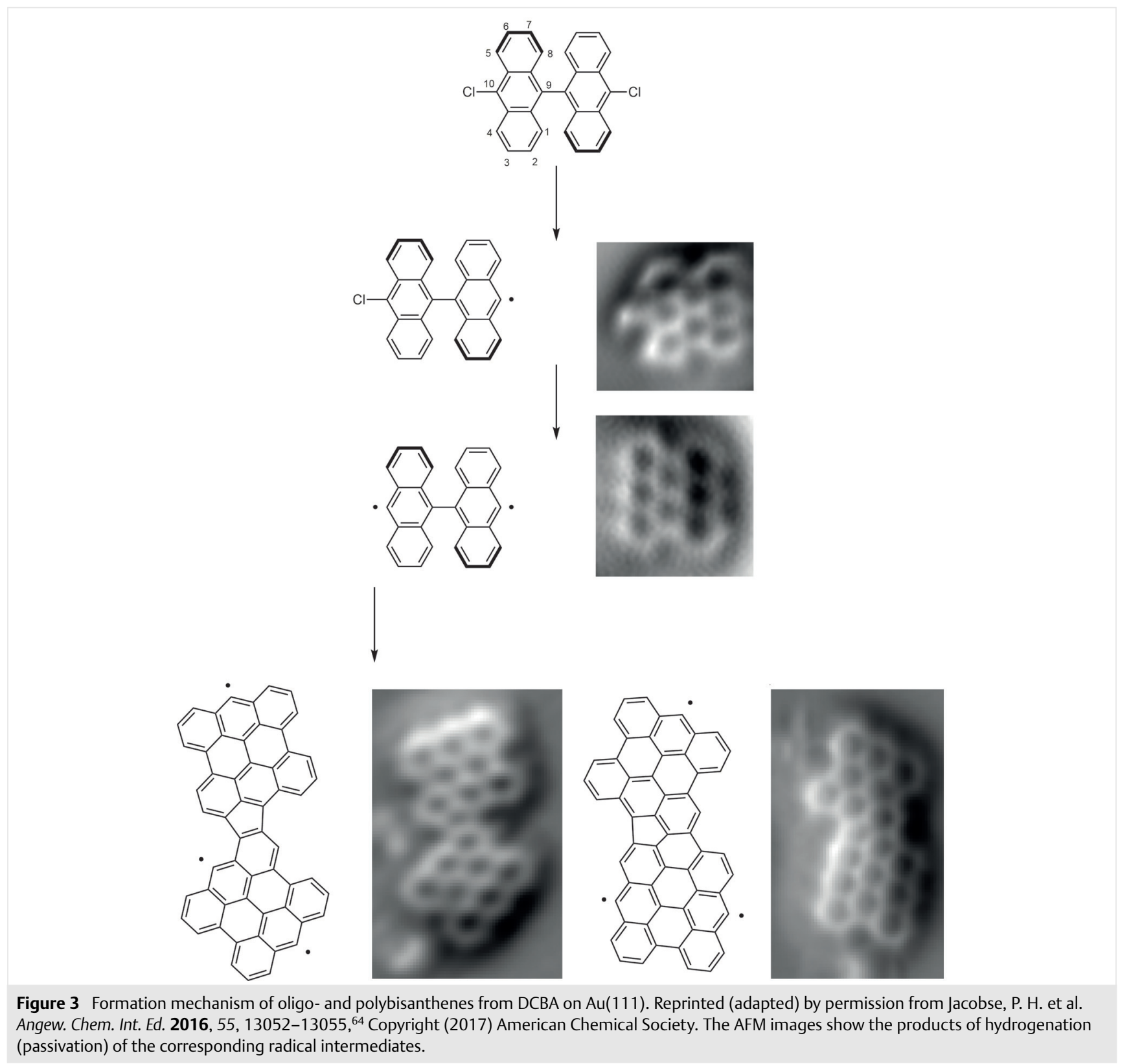

exact same way as on $\mathrm{Au}(111)$. However, in STM the edges of the ribbons were found to exhibit a notable sawtoothlike appearance. This peculiar feature was initially ignored by Simonov et al., ${ }^{66}$ but at the same time prompted Han et al. to claim the product to be the counterintuitive 3,1-chiral GNR (see Figure 4). ${ }^{67}$ The dispute that followed between the two groups could not be settled by STM imaging. ${ }^{68-71}$

In order to solve the controversy, we started by growing GNRs from DBBA on $\mathrm{Cu}(111)$ in the same way as Han and Simonov, and performed nc-AFM experiments to indisputably reveal the product to be the 3,1-chiral GNR. ${ }^{72} \mathrm{~A}$ simultaneous effort by Sánchez-Sánchez et al. corroborated these results. ${ }^{73}$ The inevitable conclusion is that the Ullmann coupling, which should interconnect the $\mathrm{C}(10)$ positions of bianthryls, is compromised; in contrast, the chiral nanoribbons feature bonds between the $\mathrm{CH}(2)$ positions of adjacent monomers. We continued the experiments by attempting the synthesis with both DCBA and unsubstituted bianthryl. Surprisingly, in all three cases we obtained the 3,1-chiral nanoribbon. Evidently, the halogen at the $\mathrm{C}(10)$ position does not play an important role in the reaction. Therefore, we concluded that bianthryls could be activated directly at the $\mathrm{CH}(2)$ positions by the copper surface. 

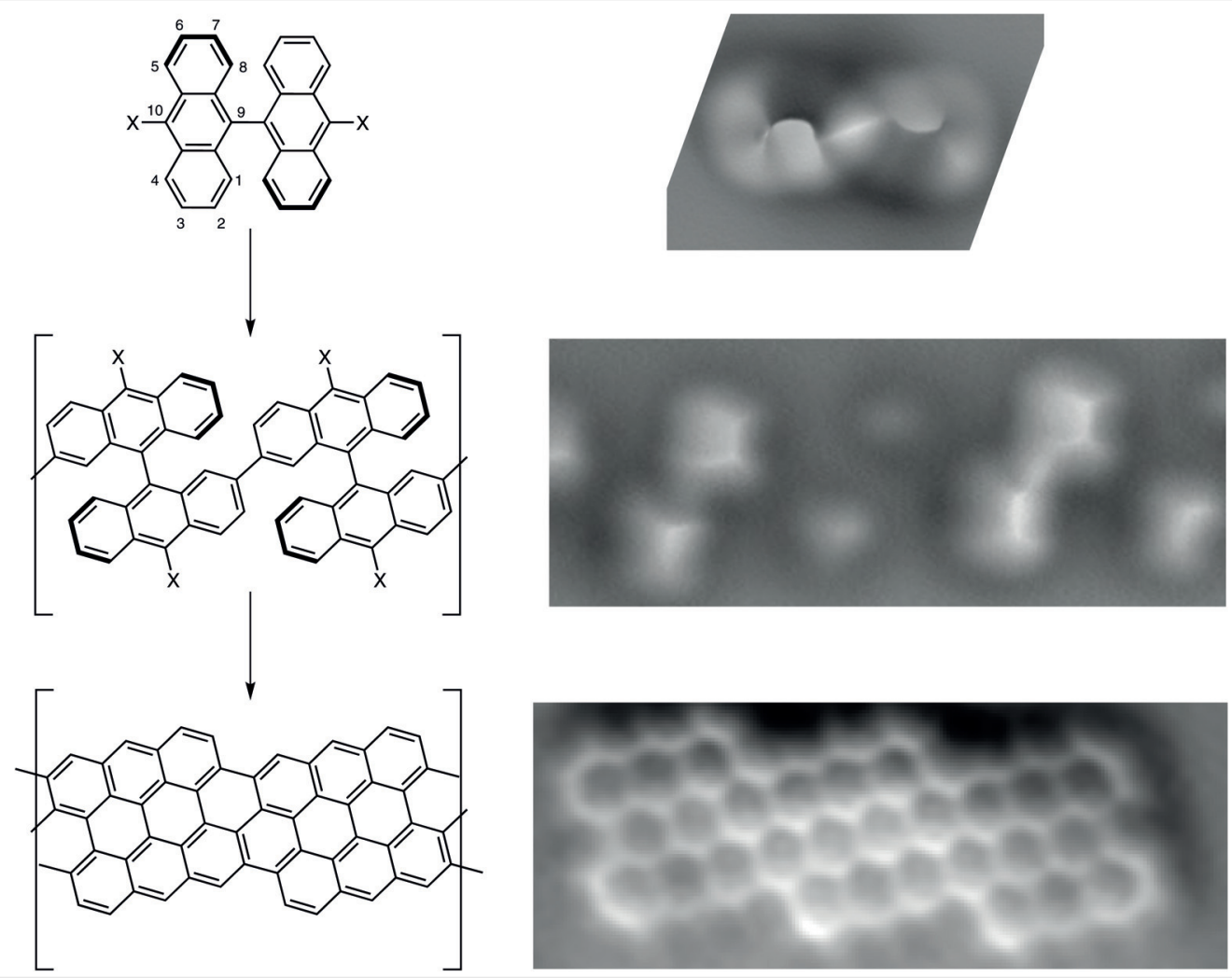

Figure 4 Formation mechanism of 3,1-chiral graphene nanoribbons on $\mathrm{Cu}(111)$. The substituent $\mathrm{X}=\mathrm{H}, \mathrm{Cl}, \mathrm{Br}$. Reprinted (adapted) by permission from Schulz, F. et al. J. Phys. Chem. C 2017, 121, 2896-2904, ${ }^{72}$ Copyright (2017) American Chemical Society.

We decided to delve more deeply into this problem by attempting to identify intermediates in the mechanism. As noted by Simonov, the GNR formation from DBBA on $\mathrm{Cu}(111)$ is a three-step process, with an additional intermediate as compared to the two-step process on $\mathrm{Au}(111)$. Instead of going through a single polyanthrylene stage - or rather its chiral GNR counterpart - two different staggered polymer structures appear in successive stages with DBBA. The first polymer, with large spacing, was thought to originate from an organometallic chain containing copper atoms in between the adjacent monomers. Unfortunately, due to the staggered nature of the chains, the hypothetical interstitial copper atoms are hidden away from view as far as ncAFM is concerned. We noted that the long-periodicity intermediate could only be obtained using DBBA and not for either DCBA or bianthryl, suggesting that the nature of the intermediate is indeed related to a debrominated product, and may therefore be the $\mathrm{C}(10)$-coupled product. By performing nc-AFM, we could determine the orientation of the monomers in both chains. This allowed us to conclude that the short-periodicity chain is the $\mathrm{CH}(2)-\mathrm{CH}(2)$ coupled polymer. The long-periodicity chain appeared to be connected in the regular Ullmann-type fashion, with extra spacing between the monomers likely originating from the copper atoms.
We completed our analysis by tip-manipulation experiments, and by looking into the analogous 5-AGNR formation from dibromoperylene. ${ }^{72}$ This reaction only involves planar products, and indeed allowed us to identify both organometallic and covalent stages in the reaction. By combining all the information, we could establish the mechanism by which the nanoribbons form on $\mathrm{Cu}(111)$. Even though DBBA debrominates and takes the first step to assemble into the usual polyanthrylene chain and subsequent 7-AGNR, the Ullmann coupling is still intercepted by activation of $\mathrm{CH}(2)$ and covalent coupling at this position. On the other hand, activation at the $\mathrm{C}(10)$ position plays no role in bianthryl nor in DCBA. In these cases, the organometallic 'Ullmann' intermediate is absent and coupling at the $\mathrm{CH}(2)$ positions produces the covalent polymer directly. Subsequent cyclodehydrogenation produces the 3,1-chiral nanoribbon in all three cases.

The elucidation of the formation mechanism of chiral nanoribbons on $\mathrm{Cu}(111)$, as well as that of the oligo- and polybisanthenes on $\mathrm{Au}(111)$ are cases where uncovering the structure of products and intermediates provides a unique insight into the processes occurring in on-surface chemistry. As nanostructures continue to grow more sophisticated and complex, nc-AFM will prove to be an im- 
portant tool to elucidate molecular frameworks of products and intermediates, providing a unique understanding that cannot be obtained with any other method.

\section{Funding Information}

We gratefully acknowledge funding by a NWO Graduate program and the Sector Plan Chemistry and Physics.

\section{References}

(1) Davy, H. Philos. Trans. R. Soc. London 1817, 107, 77.

(2) Lindner, R.; Kühnle, A. ChemPhysChem 2015, 16, 1582.

(3) Björk, J. J. Phys. Condens. Matter 2016, 28, 83002.

(4) Narita, A.; Wang, X.-Y.; Feng, X.; Müllen, K. Chem. Soc. Rev. 2015, $44,6616$.

(5) Narita, A.; Feng, X.; Müllen, K. Chem. Rec. 2015, 15, 295.

(6) Méndez, J.; López, M. F.; Martín-Gago, J. A. Chem. Soc. Rev. 2011, $40,4578$.

(7) Martin-Gago, J. A. Nat. Chem. 2011, 3, 11.

(8) Otero, G.; Biddau, G.; Sanchez-Sanchez, C.; Caillard, R.; Lopez, M. F.; Rogero, C.; Palomares, F. J.; Cabello, N.; Basanta, M. A.; Ortega, J.; Mendez, J.; Echavarren, A. M.; Perez, R.; Gomez-Lor, B.; Martin-Gago, J. A. Nature 2008, 454, 865.

(9) Chen, C. J. Introduction to Scanning Tunneling Microscopy; OUP: Oxford, 2008.

(10) Repp, J.; Meyer, G.; Stojković, S. M.; Gourdon, A.; Joachim, C. Phys. Rev. Lett. 2005, 94, 26803.

(11) Gross, L.; Mohn, F.; Moll, N.; Liljeroth, P.; Meyer, G. Science 2009, 325, 1110.

(12) Gross, L. Nat. Chem. 2011, 3, 273.

(13) Jarvis, S. P. Int. J. Mol. Sci. 2015, 16, 19936.

(14) Altman, E. I.; Baykara, M. Z.; Schwarz, U. D. Acc. Chem. Res. 2015, $48,2640$.

(15) Gross, L.; Mohn, F.; Moll, N.; Schuler, B.; Criado, A.; Guitián, E.; Peña, D.; Gourdon, A.; Meyer, G. Science 2012, 337, 1326.

(16) Kawai, S.; Sadeghi, A.; Feng, X.; Lifen, P.; Pawlak, R.; Glatzel, T.; Willand, A.; Orita, A.; Otera, J.; Goedecker, S.; Meyer, E. ACS Nano 2013, 7, 9098.

(17) Zhang, J.; Chen, P.; Yuan, B.; Ji, W.; Cheng, Z.; Qiu, X. Science 2013, 342, 611.

(18) Hämäläinen, S. K.; van der Heijden, N.; van der Lit, J.; den Hartog, S.; Liljeroth, P.; Swart, I. Phys. Rev. Lett. 2014, 113, 186102.

(19) Hapala, P.; Švec, M.; Stetsovych, O.; van der Heijden, N. J.; Ondráček, M.; van der Lit, J.; Mutombo, P.; Swart, I.; Jelínek, P. Nat. Commun. 2016, 7, 11560.

(20) Schuler, B.; Meyer, G.; Peña, D.; Mullins, O. C.; Gross, L. J. Am. Chem. Soc. 2015, 137, 9870.

(21) Lafferentz, L.; Eberhardt, V.; Dri, C.; Africh, C.; Comelli, G.; Esch, F.; Hecht, S.; Grill, L. Nat. Chem. 2012, 4, 215.

(22) Kawai, S.; Haapasilta, V.; Lindner, B. D.; Tahara, K.; Spijker, P.; Buitendijk, J. A.; Pawlak, R.; Meier, T.; Tobe, Y.; Foster, A. S.; Meyer, E. Nat. Commun. 2016, 7, 12711.

(23) Wang, D.; Chen, Q.; Wan, L.-J. Phys. Chem. Chem. Phys. 2008, 10, 6467.

(24) Hla, S.-W.; Bartels, L.; Meyer, G.; Rieder, K.-H. Phys. Rev. Lett. 2000, 85, 2777.

(25) Hla, S.-W.; Rieder, K.-H. Annu. Rev. Phys. Chem. 2003, 54, 307.

(26) Schuler, B.; Fatayer, S.; Mohn, F.; Moll, N.; Pavliček, N.; Meyer, G.; Peña, D.; Gross, L. Nat. Chem. 2016, 8, 220.
(27) Riss, A.; Paz, A. P.; Wickenburg, S.; Tsai, H.-Z.; De Oteyza, D. G.; Bradley, A. J.; Ugeda, M. M.; Gorman, P.; Jung, H. S.; Crommie, M. F.; Rubio, A.; Fischer, F. R. Nat. Chem. 2016, 8, 678.

(28) Pavliček, N.; Schuler, B.; Collazos, S.; Moll, N.; Pérez, D.; Guitián, E.; Meyer, G.; Peña, D.; Gross, L. Nat. Chem. 2015, 7, 623.

(29) de Oteyza, D. G.; Gorman, P.; Chen, Y.-C.; Wickenburg, S.; Riss, A.; Mowbray, D. J.; Etkin, G.; Pedramrazi, Z.; Tsai, H.-Z.; Rubio, A.; Crommie, M. F.; Fischer, F. R. Science 2013, 340, 1434.

(30) Albrecht, F.; Pavliček, N.; Herranz-Lancho, C.; Ruben, M.; Repp, J. J. Am. Chem. Soc. 2015, 137, 7424.

(31) Albrecht, F.; Bischoff, F.; Auwärter, W.; Barth, J. V.; Repp, J. Nano Lett. 2016, 16, 7703.

(32) Pavliček, N.; Fleury, B.; Neu, M.; Niedenführ, J.; Herranz-Lancho, C.; Ruben, M.; Repp, J. Phys. Rev. Lett. 2012, 108, 86101.

(33) Kawai, S.; Foster, A. S.; Björkman, T.; Nowakowska, S.; Björk, J.; Canova, F. F.; Gade, L. H.; Jung, T. A.; Meyer, E. Nat. Commun. 2016, 7, 11559 .

(34) He, Y.; Garnica, M.; Bischoff, F.; Ducke, J.; Bocquet, M.-L.; Batzill, M.; Auwärter, W.; Barth, J. V. Nat. Chem. 2016, 9, 1.

(35) Celis, A.; Nair, M. N.; Taleb-Ibrahimi, A.; Conrad, E. H.; Berger, C.; de Heer, W. A.; Tejeda, A. J. Phys. D. Appl. Phys. 2016, 49, 143001.

(36) Terrones, M.; Botello-Méndez, A. R.; Campos-Delgado, J.; LópezUrías, F.; Vega-Cantú, Y. I.; Rodríguez-Macías, F. J.; Elías, A. L.; Muñoz-Sandoval, E.; Cano-Márquez, A. G.; Charlier, J.-C.; Terrones, H. Nano Today 2010, 5, 351.

(37) Ezawa, M. Phys. Rev. B 2006, 73, 45432.

(38) Barone, V.; Hod, O.; Scuseria, G. E. Nano Lett. 2006, 6, 2748.

(39) Castro Neto, A. H.; Guinea, F.; Peres, N. M. R.; Novoselov, K. S.; Geim, A. K. Rev. Mod. Phys. 2009, 81, 109.

(40) Cai, J.; Pignedoli, C. A.; Talirz, L.; Ruffieux, P.; Söde, H.; Liang, L.; Meunier, V.; Berger, R.; Li, R.; Feng, X.; Müllen, K.; Fasel, R. Nat. Nanotechnol. 2014, 9, 1.

(41) Wang, X.; Ouyang, Y.; Jiao, L.; Wang, H.; Xie, L.; Wu, J.; Guo, J.; Dai, H. Nat. Nanotechnol. 2011, 6, 563.

(42) Liao, W.; Bao, H.; Guo, J.; Zhao, H. Appl. Phys. A 2015, 120, 657.

(43) Talirz, L.; Söde, H.; Dumslaff, T.; Wang, S.; Sanchez-Valencia, J. R.; Liu, J.; Shinde, P.; Pignedoli, C. A.; Liang, L.; Meunier, V.; Plumb, N. C.; Shi, M.; Feng, X.; Narita, A.; Müllen, K.; Fasel, R.; Ruffieux, P. ACS Nano 2017, 11, 1380.

(44) Söde, H.; Talirz, L.; Gröning, O.; Pignedoli, C. A.; Berger, R.; Feng, X.; Müllen, K.; Fasel, R.; Ruffieux, P. Phys. Rev. B 2015, 91, 45429.

(45) Wang, S.; Talirz, L.; Pignedoli, C. A.; Feng, X.; Müllen, K.; Fasel, R.; Ruffieux, P. Nat. Commun. 2015, 7, 11507.

(46) Cai, J.; Ruffieux, P.; Jaafar, R.; Bieri, M.; Braun, T.; Blankenburg, S.; Muoth, M.; Seitsonen, A. P.; Saleh, M.; Feng, X.; Müllen, K.; Fasel, R. Nature 2010, 466, 470.

(47) Batra, A.; Cvetko, D.; Kladnik, G.; Adak, O.; Cardoso, C.; Ferretti, A.; Prezzi, D.; Molinari, E.; Morgante, A.; Venkataraman, L. Chem. Sci. 2014, 5, 4419.

(48) Bronner, C.; Björk, J.; Tegeder, P. J. Phys. Chem. 2015, 119, 486.

(49) Björk, J.; Stafström, S.; Hanke, F. J. Am. Chem. Soc. 2011, 133, 14884.

(50) van der Lit, J.; Boneschanscher, M. P.; Vanmaekelbergh, D.; Ijäs, M.; Uppstu, A.; Ervasti, M.; Harju, A.; Liljeroth, P.; Swart, I. Nat. Commun. 2013, 4, 2023.

(51) Talirz, L.; Ruffieux, P.; Fasel, R. Adv. Mater. 2016, 28, 6222.

(52) Yang, X.; Dou, X.; Rouhanipour, A.; Zhi, L.; Räder, H. J.; Müllen, K.; Ra, H. J.; Mu, K. J. Am. Chem. Soc. 2008, 130, 4216.

(53) Narita, A.; Feng, X.; Hernandez, Y.; Jensen, S. A.; Bonn, M.; Yang, H.; Verzhbitskiy, I. A.; Casiraghi, C.; Hansen, M. R.; Koch, A. H. R.; Fytas, G.; Ivasenko, O.; Li, B.; Mali, K. S.; Balandina, T.; Mahesh, S.; De Feyter, S.; Müllen, K. Nat. Chem. 2014, 6, 126.

(54) James, D. K.; Tour, J. M. Macromol. Chem. Phys. 2012, 213, 1033. 
(55) Yazyev, O. V. Acc. Chem. Res. 2013, 46, 2319.

(56) Kawai, S.; Saito, S.; Osumi, S.; Yamaguchi, S.; Foster, A. S.; Spijker, P.; Meyer, E. Nat. Commun. 2015, 6, 8098.

(57) Bronner, C.; Stremlau, S.; Gille, M.; Brauße, F.; Haase, A.; Hecht, S.; Tegeder, P. Angew. Chem. Int. Ed. 2013, 52, 4422.

(58) Zhang, Y.; Zhang, Y.; Li, G.; Lu, J.; Lin, X.; Du, S.; Berger, R.; Feng, X.; Müllen, K.; Gao, H.-J. Appl. Phys. Lett. 2014, 105, 23101.

(59) Cai, J.; Pignedoli, C. A.; Talirz, L.; Ruffieux, P.; Söde, H.; Liang, L.; Meunier, V.; Berger, R.; Li, R.; Feng, X.; Müllen, K.; Fasel, R. Nat. Nanotechnol. 2014, 9, 896.

(60) Cloke, R. R.; Marangoni, T.; Nguyen, G. D.; Joshi, T.; Rizzo, D. J.; Bronner, C.; Cao, T.; Louie, S. G.; Crommie, M. F.; Fischer, F. R. J. Am. Chem. Soc. 2015, 137, 8872.

(61) Nguyen, G. D.; Toma, F. M.; Cao, T.; Pedramrazi, Z.; Chen, C.; Rizzo, D. J.; Joshi, T.; Bronner, C.; Chen, Y.-C.; Favaro, M.; Louie, S. G.; Fischer, F. R.; Crommie, M. F. J. Phys. Chem. C 2016, 120, 2684.

(62) Kimouche, A.; Ervasti, M. M.; Drost, R.; Halonen, S.; Harju, A.; Joensuu, P. M.; Sainio, J.; Liljeroth, P. Nat. Commun. 2015, 6, 10177.

(63) Liu, J.; Li, B.-W.; Tan, Y.; Giannakopoulos, A.; Sanchez-Sanchez, C.; Beljonne, D.; Ruffieux, P.; Fasel, R.; Feng, X.; Müllen, K. J. Am. Chem. Soc. 2015, 137, 6097.

(64) Jacobse, P. H.; van den Hoogenband, A.; Moret, M. E.; Klein Gebbink, R. J. M.; Swart, I. Angew. Chem. Int. Ed. 2016, 55, 13052.

(65) Chen, Y.-C.; de Oteyza, D. G.; Pedramrazi, Z.; Chen, C.; Fischer, F. R.; Crommie, M. F. ACS Nano 2013, 7, 6123.
(66) Simonov, K. A.; Vinogradov, N. A.; Vinogradov, A. S.; Generalov, A. V.; Zagrebina, E. M.; Mårtensson, N.; Cafolla, A. A.; Carpy, T.; Cunniffe, J. P.; Preobrajenski, A. B. J. Phys. Chem. C 2014, 118, 12532.

(67) Han, P.; Akagi, K.; Federici Canova, F.; Mutoh, H.; Shiraki, S.; Iwaya, K.; Weiss, P. S.; Asao, N.; Hitosugi, T. ACS Nano 2014, 8, 9181.

(68) Simonov, K. A.; Vinogradov, N. A.; Vinogradov, A. S.; Generalov, A. V.; Zagrebina, E. M.; Svirskiy, G. I.; Cafolla, A. A.; Carpy, T.; Cunniffe, J. P.; Taketsugu, T.; Lyalin, A.; Mårtensson, N.; Preobrajenski, A. B. ACS Nano 2015, 9, 8997.

(69) Han, P.; Akagi, K.; Federici Canova, F.; Shimizu, R.; Oguchi, H.; Shiraki, S.; Weiss, P. S.; Asao, N.; Hitosugi, T. ACS Nano 2015, 9, 12035.

(70) Simonov, K. A.; Vinogradov, N. A.; Vinogradov, A. S.; Generalov, A. V.; Zagrebina, E. M.; Mårtensson, N.; Cafolla, A. A.; Carpy, T.; Cunniffe, J. P.; Preobrajenski, A. B. ACS Nano 2015, 9, 3399.

(71) Han, P.; Akagi, K.; Federici Canova, F.; Mutoh, H.; Shiraki, S.; Iwaya, K.; Weiss, P. S.; Asao, N.; Hitosugi, T. ACS Nano 2015, 9, 3404.

(72) Schulz, F.; Jacobse, P. H.; Canova, F. F.; van der Lit, J.; Gao, D. Z.; van den Hoogenband, A.; Han, P.; Klein Gebbink, R. J. M. M.; Moret, M.-E.; Joensuu, P. M.; Swart, I.; Liljeroth, P. J. Phys. Chem. C 2017, 121, 2896.

(73) Sánchez-Sánchez, C.; Dienel, T.; Deniz, O.; Ruffieux, P.; Berger, R.; Feng, X.; Müllen, K.; Fasel, R. ACS Nano 2016, 10, 8006. 